Available online on 15.05.2020 at jddtonline.info
Open Access to Pharmaceutical and Medical Research
unrestricted non-commercial use, provided the original work is properly cited

Open $\odot$ Access

Research Article

\title{
Vimentin and Cytokeratin Immunostaining: The Role in Basic Diagnosis and Prognosis of Sarcomas
}

\author{
Samuel Ifedioranma Ogenyia*, Jonathan Madukweb, Anulika Obianuju Onyemelukwec, Anthony Ajuluchukwu Ngokerea \\ a* Department of Medical Laboratory Science, Nnamdi Azikiwe University, Nnewi Campus, Nigeria \\ b Department of Histopathology, National Hospital, Abuja, Nigeria \\ c Department of Medical Laboratory Science, University of Nigeria, Enugu Campus, Nigeria
}

\begin{abstract}
Introduction: Specific diagnosis which provides diagnostic, prognostic, and therapeutic information to guide patient care defines the primary goal of sarcoma management and care. The role of immunohistochemistry, using vimentin (mesenchymal linage marker) and cytokeratin (epithelial linage marker) as basic markers for diagnosis and classification of sarcomas for specific management strategies and prognosis was elucidated in the present study.

Materials and Methods: Twenty four (24) archived paraffin wax processed tissue block sarcoma samples were randomly selected from the histopathology Laboratories and museums of the Nnamdi Azikiwe University Teaching Hospital (NAUTH) Nnewi and National hospital Abuja and necessary data obtained from records. Blocks were re-embedded with fresh paraffin wax and $3 \mu$ thick sections cut with the aid of a rotary microtome. Haematoxylin and Eosin staining method was employed to confirm diagnosis before proceeding to immunohistochemistry. Antibodies for vimentin and pancytokeratin were employed for immunohistochemistry while exposed mouse and rabbit specific horseradish peroxides/diaminobenzidine detection IHC kit was employed for immunostaining.

Results: The mean age of patients was 26 years while the ages range from 11 to 48 years with 14 (58.3\%) females and 10 (41.7\%) males. Vimentin had strong positive immunoreactivity for all sarcoma samples whereas cytokeratin had positive immunoreactions for synovial sarcoma only, which also showed co-expression of both genes.

Conclusion: Vimentin and cytokeratin may play vital role as basic biomarkers not only for diagnosis and characterization of sarcomas but for specific management regime and prognostication. However, IHC must be performed at high standard using appropriate antibodies, samples and reagents.
\end{abstract}

Keywords: Sarcoma, immunohistochemistry, vimentin, cytokeratin, biomarker.

Article Info: Received 08 March 2020; Review Completed 21 April 2020; $\quad$ Accepted 01 May $2020 ; \quad$ Available online 15 May 2020

Cite this article as:

Ogenyi SI, Madukwe J, Onyemelukwe AO, Ngokere AA, Vimentin and Cytokeratin Immunostaining: The Role in Basic Diagnosis and Prognosis of Sarcomas, Journal of Drug Delivery and Therapeutics. 2020; 10(3):175-178 http://dx.doi.org/10.22270/jddt.v10i3.4033

*Address for Correspondence:

Ogenyi, Samuel Ifedioranma, Department of Medical Laboratory Science, Nnamdi Azikiwe University, Nnewi Campus, Nnewi, Anambra State, Postcode: 435101, Nigeria

\subsection{INTRODUCTION}

Vimentin is a gene which encodes for type III intermediate filament protein. Intermediate filaments, along with microtubules and actin microfilaments constitute the cytoskeleton. It has a role in neuritogenesis and cholesterol transport and functions as an organizer of a number of other critical proteins involved in cell attachment, migration, and signaling with its mutations associated with congenital cataracts in human patients. Vimentin expressed in certain types of carcinomas (renal cell carcinoma, spindle cell carcinoma), as well as lymphomas and melanomas It is also

ISSN: 2250-1177 is expressed in mesenchymal cells though, not specific for them ${ }^{1}$. Due the crucial role of vimentin in maintaining muscle cyto-architecture, it is considered an essential marker for muscle regeneration ${ }^{2}$. Cytokeratins are members of the keratin gene family and consists of basic or neutral proteins arranged in pairs of heterotypic keratin chains and co-expressed during differentiation of simple and stratified epithelial tissues. According to Wei et al ${ }^{1}$, they are proteins of keratin-containing intermediate filaments found in the intracytoplasmic cytoskeleton of epithelial tissue. They are specifically expressed in the simple epithelia lining the cavities of the internal organs and in the gland ducts and 
blood vessels and therefore, are basic makers for carcinomas. Due to the expression pattern of vimentin and cytokeratins in cancers, their immuno-reactivity pattern play a vital role in basic characterization of neoplasms into carcinomas and sarcomas 2, 3, 4, 5. It is noteworthy however, that both genes have their pitfalls as biomarkers of characterization of sarcomas and carcinomas. Wei et al ${ }^{1}$ in a review report on soft tissue immunohistochemistry update noted that vimentin may be expressed in certain types of carcinomas and may not be expressed in some mesenchymal tumours. The authors however, opined that if the mesenchymal tissue is negative for vimentin, it may indicate that the tissue is not of a mesenchymal differentiation. Cytokeratin, similarly may be expressed in some sarcomas especially epitheloid sarcomas 2,4 . Vimentin, though, cannot solely be used to differentiate mesenchymal from non mesenchymal neoplasms, the role as screening marker for neoplasms of mesenchymal linage cannot be emphasized ${ }^{4}$. The authors further noted frequent co-expression of vimentin and cytokeratin in some carcinomas which may suggest certain types of epithelial tumors as possible primary sites in the evaluation of metastatic tumors.

Sarcomas are connective tissues malignant neoplasms usually found in the skeletal muscle, fat, tendons, fibrous tissue, smooth muscle and the neurovascular elements that support these components and in bone with diverse and varied histogenesis 6 . Though, believed to account for $1 \%$ of all human malignancies, the growing incidence, the very aggressive and invasive nature and the propensity of most sarcomas to metastasize has brought it to limelight as a major health challenge 6 . Sarcomas have predilections for children and young adults with a male to female ratio of 2:17, 8. Dermatofibrosarcoma protuberans, malignant peripheral nerve sheath tumour, osteoblastic osteosarcoma, fibromyxoma, malignant mesenchymal tumour, fibromyxoma, alveolar rhabdomyosarcoma, metastatic liposarcoma, synovial sarcoma, low grade leiomyosarcoma to mention but a few are examples of most commonly diagnosed sarcoma cases in Nigeria 8 . The wide heterogeneous nature of sarcomas, with greater than 100 histological types and subtypes with considerable morphological overlaps between the different diagnostic entities ${ }^{5}$ makes it very challenging in establishing definitive diagnosis of most sarcoma types. Furthermore, sarcomas assume poorly differentiated, undifferentiated with no obvious line of differentiation or may even form as secondary implant (metastatic tumours) in distant sites, thereby increasing the challenge of definitive diagnosis. According to Wei et al ${ }^{1}$ the general approach to reaching a definitive differential diagnosis of soft tissue tumors is to first consider clinicoradiologic, histomorphologic, and cytomorphologic features of the tumour. Tumours with obvious line of differentiation such as smooth-muscle, skeletal-muscle, vascular, neural, and Chondro-osseous lineages may be diagnosed with morphological features. Contrariwise however, immunostaining, using appropriate marker will be required to actualize diagnosis.

In order actualize definitive and specific diagnosis that provides diagnostic, prognostic, and therapeutic information to guide patient care, immunohistochemistry play a crucial role 1. The role of immunohistochemical expressions of vimentin and cytokeratin, as afore stated, in the basic classification of sarcomas cannot be overemphasized. The present study aimed at evaluating the immunohistochemical expression pattern of vimentin and cytokeratin using selected samples of commonly diagnosed sarcomas in our facility. The expression pattern among the implications will highlight the role of the genes in basic characterization of sarcomas which is a basic a critical step towards patients' care and management especially in a middle income country like Nigeria.

\subsection{MATERIALS AND METHOD}

\subsection{Study area/ Study design}

This was a cross sectional study using 24 archived samples of already diagnosed sarcoma which was carried out at the Nnamdi Azikiwe University Teaching Hospital, Nnewi and National hospital Abuja.

\subsection{Ethical approval}

Ethical approval to carry out this study was obtained from the Ethical Committee of Nnamdi Azikiwe University Teaching Hospital (NAUTH) Nnewi.

\subsection{Sample collection}

Twenty four (24) archived paraffin wax processed tissue block sarcoma samples were randomly selected from the histopathology Laboratories and museums of the Nnamdi Azikiwe University Teaching Hospital (NAUTH) Nnewi and National hospital Abuja. Necessary data were obtained from clinical records, operation notes and histopathology reports of the patients.

\subsection{Tissue preparation}

Tissue blocks were re-embedded in fresh paraffin wax, $3 \mu$ thick sections cut with the aid of a rotary microtome, cut sections floated out on a lukewarm Leica water bath, mounted on slides previously coated with poly-l-lysine, drained, labelled and placed on Leica hot plate in order to dry and affix the tissue onto the slides.

\subsection{Staining}

Cut Sections were stained by Haematoxylin and Eosin (H\&E) method and morphological diagnosis of each sample confirmed before proceeding to immunostaining.

\subsection{Immunohistochemical Staining (IHC)}

IHC of test materials and positive controls were carried according to a method by Nishio et al ${ }^{9}$. Antibodies for vimentin and pancytokeratin were employed for immunohistochemistry. Exposed Mouse and Rabbit Specific horseradish peroxidase/diaminobenzidine (HRP/DAB) detection IHC kit was employed for immunostaining while detection of immunoreactivity was performed according to manufacturer's instruction. Both antibodies and detection kits were procured from Abcam Plc Cambridge UK.

\subsection{Immunoreactivity Scoring}

Immunoreactivity was semi-quantitatively scored according to Zlobec et al 10. This was based on percentage of cells (area) that will stain positive and the intensity of the staining (strong, moderate, weak). A score of $5+$ was assigned to $80 \%$ or more of epithelial and/ or stromal cells that stained positive with strong intensity, 4+ was assigned to $50 \%$ or more (but less than $80 \%$ ) of epithelial cells and/ stromal cells with strong intensity or $80 \%$ of cells or more with moderate to weak intensity; $3+$ was assigned to $30 \%$ or more of epithelial and/ or stromal cells with strong intensity or $50 \%$ or more (but less than $80 \%$ ) of positive cells with moderate to weak intensity; $2+$ was assigned to $10 \%$ or more cells that stained positive with strong intensity or $30 \%$ or more ( but less than $50 \%$ ) that stained moderate to weak and $1+$ was assigned to $10 \%$ or more cells (less than $30 \%$ of positive cells) that stained positive with moderate to weak intensity; 0 was assigned to less than $10 \%$ of positivity irrespective of the intensity of staining. 


\subsection{Data Analyses}

Numerical data were summarized using mean and standard deviation, whereas categorical data were being presented using frequency and proportion. Immunoreactivity pattern was expressed as percentages.

\subsection{RESULTS}

The mean age of patients was 26 years while the ages range from 11 to 48 years with $14(58.3 \%)$ females and 10 $(41.7 \%)$ males. The breakdown of sarcoma samples were as follows: 4 dermatofibrosarcoma protuberans, 4 malignant peripheral nerve sheath tumour, 4 fibromyxoma and 2 each of osteoblastic osteosarcoma, malignant mesenchymal tumour, alveolar rhabdomyosarcoma, metastatic liposarcoma, synovial sarcoma and low grade leiomyosarcoma. Histopathological and immunoreactivity features for vimentin and cytokeratin were as shown in Table 1. Vimentin had strong positive immunoreactivity for all sarcoma samples where as cytokeratin had positive immunoreactions for synovial sarcoma only, which also showed co-expression of both genes Photomicrographs of immunohistochemical staining pattern of anti vementin and cytokeratin sarcoma samples are as shown in (Figure 1).

Table 1: Histopathological and immunoreactivity features for vimentin and cytokeratin

\begin{tabular}{|l|l|l|l|}
\hline Tumour type & No of samples & Vimentin & Cytokeratin \\
\hline Dermatofibrosarcoma protuberans & 4 & $4(100 \%) 4+(2), 5+(2)$ & $0(0 \%)$ \\
\hline Malignant peripheral nerve sheath tumour & 4 & $4(100 \%) 5+(4)$ & $0(0 \%)$ \\
\hline Osteoblastic osteosarcoma & 2 & $4(100 \%) 4+(2)$ & $0(0 \%)$ \\
\hline Fibromyxoma & 4 & $4(100 \%) 5+(4)$ & $0(0 \%)$ \\
\hline Malignant mesenchymal tumour & 2 & $2(100 \%) 5+(2)$ & $0(0 \%)$ \\
\hline Alveolar rhabdomyosarcoma & 2 & $2(100 \%) 5+(2)$ & $0(0 \%)$ \\
\hline Metastatic liposarcoma & 2 & $2(100 \%) 5+(2)$ & $0(0 \%)$ \\
\hline Synovial sarcoma & 2 & $2(100 \%) 5+(2)$ & $0(0 \%)$ \\
\hline Low grade leiomyosarcoma & 2 & $2(100 \%) 5+(2)$ & $2(100 \%) 3+(2)$ \\
\hline
\end{tabular}

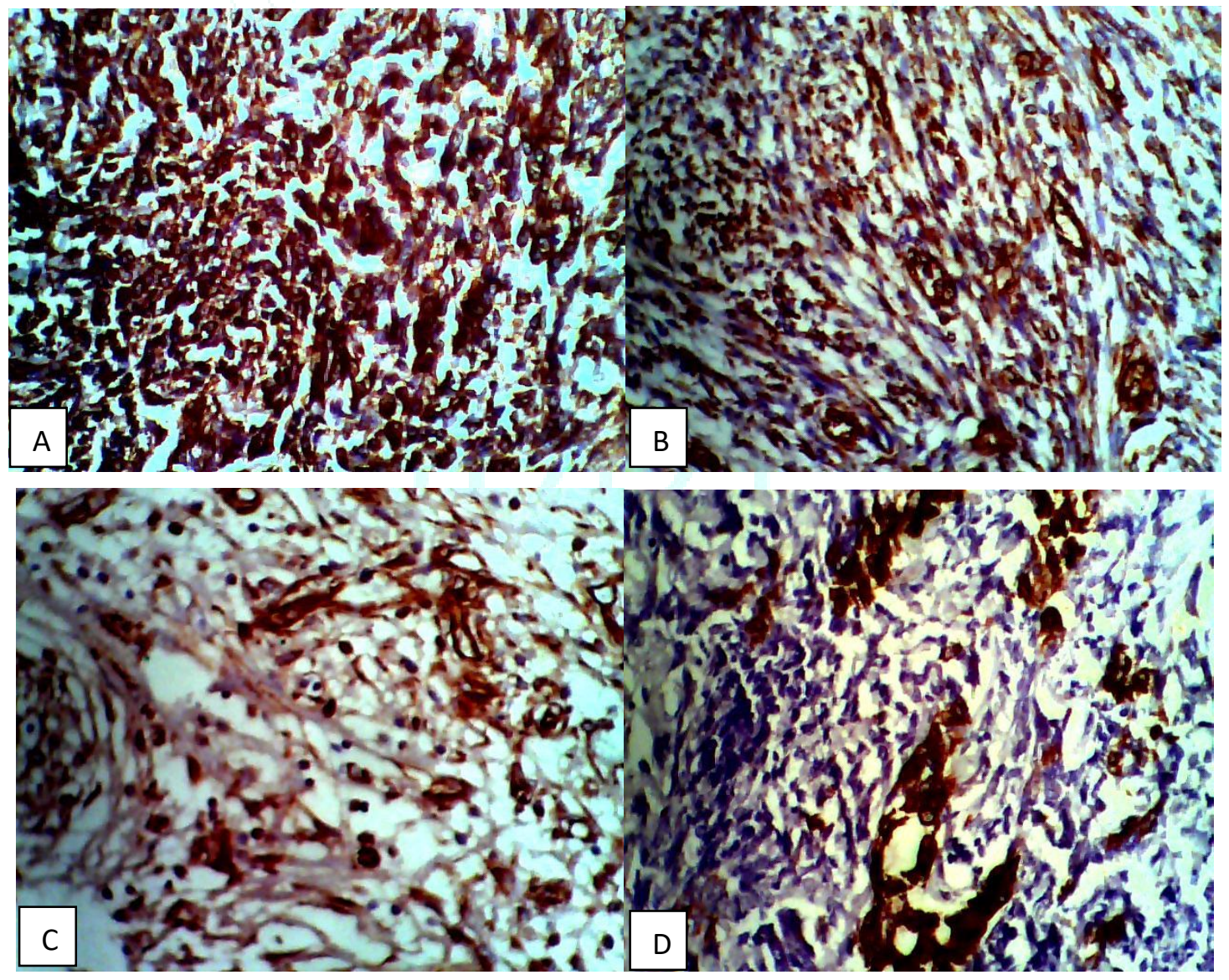

Figure 1: Immunohistochemical staining pattern of anti vimentin and cytokeratin

A: Strongly positive vimentin staining for low synovial sarcoma (X400), B: Strongly positive vimentin staining for low grade leiomyosarcoma (X400), C: Strongly positive vimentin staining for fibromyxoma (X400), D: Focally positive cytokeratin staining for synovial sarcoma (X400). 


\subsection{DISCUSSION}

The significance role of IHC expression features of vimentin and cytokeratin in characterization of sarcoma have been reported $1,3,4,5$. The present study reported 26 years as the mean age of patients, while the ages range from 11 to 48 years with a male to female ratio of 1:1.4, thus agreeing with the finding of Ikeri et al 11 who reported a median age of 33 years and a male to female ratio of 1:1.3 in an earlier study on the histological types of soft-tissue sarcomas at the Lagos University Teaching Hospital but differs from that of Dauda et al 8 and Mandong et al 7 who reported male to female ratio of 2:1 in a similar but separate studies. The authors however, corroborated the present study on the most commonly affected age ranges and the most commonly diagnosed sarcomas in Nigeria. The sight deviation from male to female ratio though not very significant could be a function of time and sample size.

Strongly positive vimentin immonoreactivity was reported for all sarcoma samples and negative cytokeratin expression except for synovial sarcoma. This agrees with earlier studies. Bashyal et al ${ }^{3}$ reported positive vimentin immunoreactivity and negative cytokeratin IHC staining for most round cell malignant sarcomas, which led them to conclude that IHC provide important tool for clear distinction between tumours. This finding not only highlights the need for inclusion of IHC as an important ancillary diagnostic tool but strongly support the application of vimentin and keratin IHC for both diagnosis and characterization of sarcomas. Suffice it to note once again that sarcomas with their characteristic divergent histogenesis with the attendant morphological presentations most times cannot be granted definitive diagnosis using only morphological appearance, hence the need for IHC. Vimentin and keratin which are known markers of mesenchymal and epithelial linage respectively present an indispensible tool in characterization of sarcomas especially when the linage is not obvious. Despite the fact that Wei et al ${ }^{1}$ opined that vimentin positivity has a limited value in the diagnosis of soft tissue tumors, the authors however, accepted its role in establishing that a tumour is of mesenchymal differentiation and not of epithelial differentiation. The co-expression of vimentin and keratin in synoval sarcoma could be explained by the nature of the tumour in question. According to Morgan ${ }^{1}$, synoval sarcoma may either be biphasic (with both mesenchymal and epithelial components present) or monophasic (with either mesenchymal or epithelial morphology) histologically. Histologically biphasic tumour show positive immunoreactivity for both vimentin and cytokeratin while monophasic type show immunoraectivity for either of the genes depending on their linage. This fact further established the role of the markers not only for diagnosis and characterization but for determination specific therapy/management options towards better patients' care and treatment. Besides the application of vimentin and cytokeratin IHC in diagnosis and characterization of sarcomas it aids their management and prognosis. This was the view of Parham 5 in his reported on the imperative of IHC and its potential applications, not just for diagnosis and prognostication, but for personalized therapy decisions. The strong positivity of vimentin in all sarcoma samples studied not only agreed with previous studies but validates its presence in mesenchymal tumours.

\subsection{CONCLUSION}

It could be concluded from the finding of the present study that vimentin and cytokeratin may play vital role as basic biomarkers not only for diagnosis and characterization of sarcomas but for specific management regime and prognostication. However, IHC must be performed at high standard using appropriate antibodies, samples and reagents.

\section{CONFLICTS OF INTEREST}

The authors declare that there are no conflicts of interest

\section{ACKNOWLEDGEMENTS}

The authors wish to express their immense gratitude to Tertiary Education Trust fund (TET fund) Nigeria for funding this study. Our profound gratitude also goes to the staff of Histopathology Department, Nnamdi Azikiwe University Teaching Hospital Nnewi and National Hospital Abuja for allowing us access to sample and data.

\section{REFERENCES}

1. Wei S, Henderson-Jackson E Qian X, Bui MM, "Soft Tissue Tumor Immunohistochemistry Update" Arch Pathol Lab Med, 2017; 141:1072-1091.

2. Soglia F, Mazzoni M, Zappaterra M, Di Nunzio M, Babini E, Bordini M, Sirri F, Clavenzani P, Davoli R, Petracci M, "Distribution and Expression of Vimentin and Desmin in Broiler Pectoralis major Affected by the Growth-Related Muscular Abnormalities" Front. Physiol, 2020; 10:1581.

3. Bashya R, Pathak TB, Shrestha S, Pun CB, Banstola S, Neupane $\mathrm{S}$, Lee MC, "Role of immunohistochemistry in the diagnosis of malignant small round cell tumors" JPN, 2011; 1:87-91

4. Bahrami A, Truong LD, Jae Y. Ro JY, "Undifferentiated Tumor: True Identity by Immunohistochemistry" Arch Pathol Lab Med, 2008; 132:326-348

5. Parham DM, "Immunohistochemical Markers of Soft Tissue Tumors: Pathologic Diagnosis, Genetic Contributions, and Therapeutic Options" Anal. Chem. Insights, 2015; 10(S1):1-10.

6. Kristin B, Sean D, Cristina RA, Ursula LH, Robert LW, Yidong C, Arthur AG, Paul HD, Paul SM, "Gene Expression Profiling of Human Sarcomas: Insights into Sarcoma Biology" Cancer Res, 2005; 65(20)9226- 35.

7. Mandong BM, Kidmas AT, Manasseh AN, Echejoh GO, Tanko MN, Madaki AJ, "Epidemiology of soft tissue sarcomas in Jos, North Central Nigeria" Niger J Med. 2007; 16(3):246-9.

8. Dauda MA, Yakubu D, Mandong BM, Ojo EO, "Sarcomas in Nigerian Children in Jos North Central Nigeria" Afr J Med Med Sci, 2014; 43(Suppl 1):37-44.

9. Nishio J, Althof, PA, Bailey JM, Zhou M, Neff JR, Barr FG, Parham DM, Teot L, Qualman SJ, Bridge JA, "Use of a novel FISH assay on paraffin-embedded tissues as an adjunct to diagnosis of alveolar rhabdomyosarcoma" Lab. Invest, 2006; 86:547-556.

10. Zlobec I, Steel R, Michel RP, Compton CC, Lugli A, Jass JR, "Scoring of p53, VEGF, Bcl-2 and APAF-1 immunohistochemistry and interobserver reliability in colorectal cancer" Mod Patholo, 2006; 19:1236-1242.

11. Ikeri NZ, Akinjo AO, Ajayi 00, Fehintola Banjo AA, Histological types of soft-tissue sarcomas at the Lagos university teaching hospital" Niger Postgrad Med J, 2017; 24:205-9.

12. Morgan R, Handbook of Small Animal Practice. $5^{\text {th }}$ ed. Philadelphia: W.B. Saunders Ltd; 2008, P, 763-777 